\title{
Design of a Modular and Orientable Electrodynamic Shield for Lunar Dust Mitigation
}

\author{
Malcolm Tisdale ${ }^{1,3}$, Isabella Dulá1,3, Luis A. Pabon Madrid ${ }^{1,3}$, Polina \\ Verkhovodova ${ }^{1,4}$, Jules Pénot ${ }^{1,4}$, Kaila Coimbra ${ }^{1,4}$, Leah S. Soldner ${ }^{1,4}$, \\ Tanmay Gupta ${ }^{1,4}$, Rithvik Musuku ${ }^{1,4}$, and Soon-Jo Chung ${ }^{2}$ \\ California Institute of Technology, Pasadena, CA 91125, United States
}

\begin{abstract}
Lunar dust is considered one of the main limiting factors for lunar missions of extended duration and the establishment of a sustainable human presence on the moon. The dust is difficult to shield against and remove as it is extremely abrasive, highly cohesive, small, and may be electrostatically charged. In addition to threatening astronaut health, lunar dust issues have also resulted in incorrect instrument readings, vision and optical system obscuration, performance reduction, altered thermal properties, and equipment failure. To address these issues, this paper introduces the Habitat Orientable and Modular Electrodynamic Shield (HOMES). HOMES is a collection of $0.25 \mathrm{~m} \times 0.25 \mathrm{~m}$ and $1.1 \mathrm{~kg}$ panels requiring 8 watts of power, embedded with Electrodynamic Dust Shielding (EDS) to mitigate lunar dust in a variety of applications. The modular design of HOMES addresses the gap in scalability of current EDS implementations. The EDS within HOMES is a series of 3-phase wire electrodes that induce a traveling wave-like electric field to locomote dust particles in a fixed direction. The fringing electric field generated by the EDS can keep dust particles off spacesuits and nearby objects. HOMES is a scalable modular system with rotationally symmetric panels that can be tiled to fit the desired use case. The panels are orientable to allow for customizable field directions. Among other applications, HOMES can be used to create dust free and self-cleaning work surfaces, floors, and doormats. This technology advances the state-of-the-art by incorporating EDS in a lightweight, modular design that will allow for flexibility in lunar dust mitigation within the context of the Artemis missions and beyond. The paper is divided into the following sections: problem statement and background, where the lunar dust problem and the current state of EDS technology are introduced; project description, where project requirements and design assumptions are detailed; mechanical design, outlining the EDS enclosure capable of sustaining the extreme lunar environment and an astronaut's weight; electrical design, detailing the layout of electrodes and EDS electronics; verification and validation, plan to bring homes to Technological Readiness Level 6; path to flight, discussing the necessary steps to ready HOMES for the moon. Having been awarded funding through NASA's BIG Idea Challenge, future work will focus on the construction, testing, and experimental results of this novel technology.
\end{abstract}

\footnotetext{
${ }^{1}$ Student, Department of Mechanical Engineering, AIAA Student Member.

${ }^{2}$ Bren Professor of Aerospace; Jet Propulsion Laboratory Research Scientist, Graduate Aerospace Laboratories of the California Institute of Technology (GALCIT), AIAA Associate Fellow.

${ }^{3}$ Contributed equally to the paper as First Author.

${ }^{4}$ Contributed equally to the paper as Second Author.
} 


\section{Nomenclature}

$\begin{array}{cl}\alpha & =\text { adhesion constant } \\ E & =\text { electric field } \\ \epsilon_{0} & =\text { permittivity of free space } \\ F_{D} & =\text { dielectropheric force } \\ F_{E} & =\text { electrostatic force } \\ K(\omega) & =\text { Clausius-Mossotti function } \\ q & =\text { particle charge } \\ R & =\text { particle radius }\end{array}$

\section{Introduction}

As humans seek to establish a permanent presence on the moon, the effects of lunar dust on the astronauts and equipment must be addressed. Astronauts on the Apollo missions experienced issues with dust adhesion and abrasion, leading to mechanical failure and adverse health effects due to the ingress of dust in the lunar lander. Exacerbated by the fact that the dust is electrically charged, the inhalation of these lunar dust particles could cause chronic respiratory problems as well as other unforeseen adverse effects [1]. A significant portion of the dust is collected in the lower regions of the spacesuit due to dust plumes being kicked up as the astronaut walks on the lunar surface. Furthermore, the abrasive effect of the dust increases the wear and tear of surface equipment, thereby reducing their lifetime [1]. The jagged and charged nature of lunar dust particles offers significant risk to the function of mechanical systems and the health of astronauts. A Dust Mitigation Gap Assessment Report [2] published by an International Working Group of space administrations across the world, including NASA, reported on the various approaches that could aid in dust mitigation. According to the assessment, there are three methodologies with which to mitigate dust: fluidal, mechanical, and electrodynamic/electrostatic. Fluidal solutions, though potentially effective, usually depend on consumables that decrease the long-term viability of these methods. Mechanical solutions have reduced efficacy because of the charged nature of the particles [2]. This leaves electrodynamic and electrostatic solutions as the most promising avenue for technology development to mitigate lunar dust. One such technology is electrodynamic dust shielding (EDS), which is elaborated upon in the next section. Potential stakeholders for a technology that leverages EDS for lunar dust mitigation includes NASA and private aerospace companies. If successful, there could also be potential terrestrial applications of HOMES. For instance, HOMES could be utilized in clean rooms to aid in research and technology development.

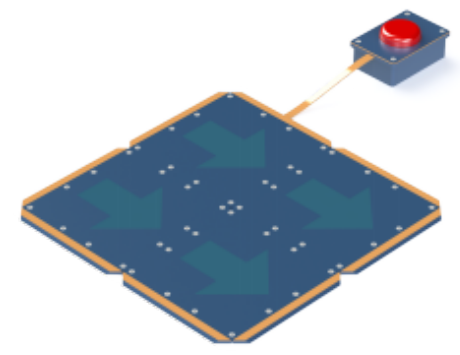

Fig. 1 Isometric view showing an example of a four-by-four configuration of HOMES, including the connected electronics box.

\section{Electrodynamic Dust Shielding Technology}

\section{A. Background}

EDS was conceived in the 1970s as a potential solution to the many challenges presented by lunar dust. Since then, the implementation of EDS has expanded to a variety of practical applications. It is still an active area of research with regards to dust mitigation designs on Lunar, Martian, and Terrestrial environments [3]. Typical implementations of EDS involve electrodes embedded in a surface that function as an interdigitated capacitor and create fringing electric fields above the surface. EDS systems work through lifting and transporting both charged 
and uncharged particles using forces generated by fringing electric fields. In particle size regimes of 20 to $500 \mu \mathrm{m}$, Coulombic forces dominate over other forces, such as adhesive and gravitational forces [4]. Thus, an EDS can successfully remove and transport particles within this regime.

EDS systems are designed to work on small scale particles [5]. In earth's atmosphere, Guo et al. showed that EDS can clear up to $90 \%$ of dust from solar panels, on timescales of around 30 seconds [6]. Similar experiments were conducted using a lunar dust simulant, JSC-1A, where $20 \mathrm{mg}$ of dust was deposited on solar panels with various electrode configurations. The solar panels operated at $20 \%$ capacity of the panel's original output voltage without any dust mitigation, but after two minutes of EDS activation, the capacity returned to $90 \%$. After 30 minutes, the capacity of the solar panels reached $98 \%$ of the original voltage output [7]. EDS has also been tested in various reduced gravity and vacuum environments. For instance, experiments have been conducted both in highvacuum and at lunar gravity using NASA's Reduced Gravity Flight aircraft. These experiments were conducted using various particle size regimes, from $10 \mu \mathrm{m}$ up to $450 \mu \mathrm{m}$, using lunar dust samples from Apollo 16. The results of the experiments proved successful, offering a promising outlook on the efficacy of dust shielding technology in the lunar environment [8]. EDS has been deployed on the International Space Station as a part of the ongoing MISSE-11 payload to understand the performance of EDS in a vacuum environment [5]. The extensive experimentation conducted with EDS has brought the base technology into TRL 8 [9]. Therefore, EDS offers an effective solution to dust mitigation. However, EDS has yet to be implemented in a modular and rugged package that could expand its use cases and versatility. The proven efficacy of EDS shows that this new avenue of implementation could have substantial success.

\section{B. Physics}

Most EDS systems employ a linear geometry with the electrodes having varying voltages to generate electric fields. The sets of electrodes within an EDS system usually have two or three distinct voltages. With two voltages, a standing wave electric field is created. However, employing three distinct voltages offers the potential to supply periodically varying voltages, generating a moving electric field that can push particles in certain directions, as seen in Fig. 2.

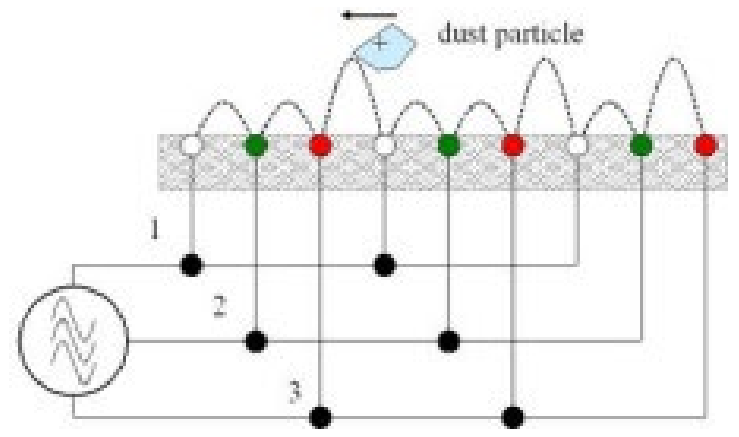

Fig. 2 An example of a three-phase EDS implementation from Calle et al. [3].

EDS makes use of electrostatic and dielectrophoretic forces to attract and transport particles. The electrostatic force is exerted on already charged dust particles. The dielectrophoretic force creates a dipole moment on particles and can be exerted on any particle regardless of charge. For an EDS system to effectively clean and remove a significant portion of the dust particles from a surface, such as the suit of an astronaut, it must overcome the Van der Waals adhesive forces and the static image charge forces. Thus, an EDS system must be designed and optimized with these forces in mind. The following equations govern the force relationships that particles feel during an implementation of EDS:

$$
\begin{gathered}
\overrightarrow{F_{E}}=q \vec{E} \\
\left\langle\overrightarrow{F_{D}}\right\rangle=2 \pi R^{3} \operatorname{Re}[\underline{K(\omega)}] \nabla \overrightarrow{E^{2}} \\
\overrightarrow{F_{V}}=\alpha R \\
\overrightarrow{F_{S}}=\frac{q}{4 \epsilon_{0}(2 R)^{2}}
\end{gathered}
$$


where Equation 1 describes the electrostatic force, Equation 2 describes the dielectrophoretic force, Equation 3 describes the Van der Waals force, and Equation 4 describes the static image charge force. An optimal EDS system should generate an electric field such that $\overrightarrow{F_{E}}$ and $\overrightarrow{F_{D}}$ can dominate over $\overrightarrow{F_{V}}$ and $\overrightarrow{F_{S}}$. Section IV, subsection $\mathrm{F}$ elaborates further on these governing equations in the implementation of a finite difference simulation to optimize and evaluate the EDS design in this proposal.

\section{Design}

Table 1 Functional requirements (FR)

\begin{tabular}{|l|l|}
\hline FR 1 & Remove 90\% of the dust in 60 seconds from each panel. \\
\hline FR 2 & Generate electric fields that are effective at attracting and transporting dust up above panel. \\
\hline FR 3 & Operate at a minimum of a 15-year lifetime on the lunar surface. \\
\hline FR 4 & Survive launch and landing loads during transport of HOMES. \\
\hline FR 5 & Support the mass of an astronaut on a single panel. \\
\hline FR 6 & Operate in lunar south pole environment conditions. \\
\hline
\end{tabular}

Table 2 Design assumptions (DA)

\begin{tabular}{|l|l|}
\hline DA 1 & Panels are placed on a level surface. \\
\hline DA 2 & $\begin{array}{l}\text { 28V DC power supplied from habitats and landers, following standards from the International } \\
\text { Space Station. }\end{array}$ \\
\hline DA 3 & Off-nominal solar activity is disregarded. \\
\hline
\end{tabular}

\section{A. Mechanical Design}

\section{Design Summary}

HOMES is designed with the objective of being an easy-to-use and modular dust mitigation system. HOMES consists of rigid EDS-embedded panels that can be attached to one another in any planar orientation to allow for different configurations and field directions. HOMES's mechanical design maximizes resilience to the lunar conditions and ease of operation while minimizing mass. The device is strong enough to sustain expected applied loads (FR 4, 5). The panels are designed to be easily carried to account for limited mobility in spacesuits. In addition, the device is designed to sustain the harsh lunar environment (FR 6). This includes varying temperature ranges and vulnerability to ionizing solar radiation. Temperatures on the lunar south pole range from approximately $-230{ }^{\circ} \mathrm{C}$ to $120^{\circ} \mathrm{C}[11]$. Thus, appropriate protections and robust materials are used to mitigate damage on the system. Lastly, this device is expected to be used for 15-30 years (FR 3). The design requirements of HOMES necessitate a material composition and structure chosen to be insensitive to temperature variances, wear over time, abrasion, and loading.

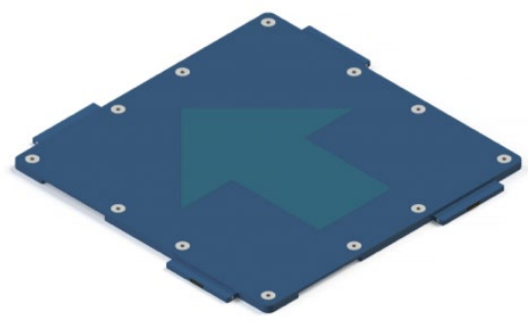

Fig. 3 Isometric view of an individual panel.

\section{Panels}

The panels were designed with simplicity and strength in mind. Each HOMES panel is square to optimize for surface area while minimizing internal bending moments and aiding with modularity (DA 1). A $0.25 \times 0.25 \mathrm{~m}$ panel size allows for significant scalability. For instance, single panels can be used for smaller tasks such as tool cleaning, a couple panels can be used to clean an astronaut's boot, and the number of panels can be increased to cover floors or walls. The panels are designed to maintain connectivity mechanically and electrically, regardless of orientation. That is, the panels can connect in any orientation to allow for customizable electric field configurations. 


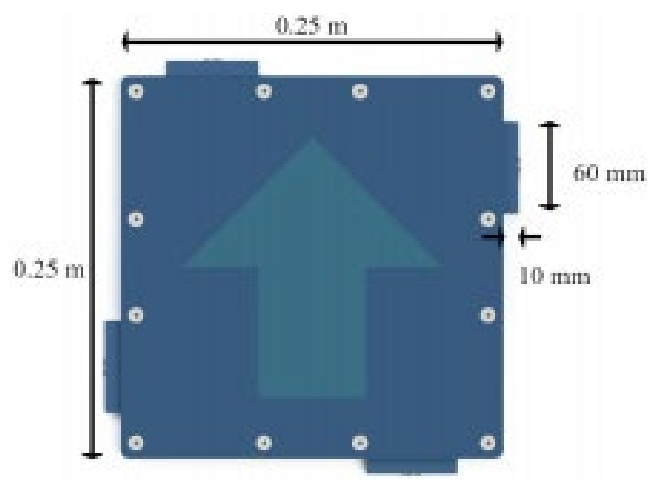

Fig. 4 Figure of individual panel with dimensions.

Each panel consists of five layers sandwiched together. The outer layers are made from Alumina, a ceramic material that will act as an electrical insulator for the device. The bottom Alumina layer is a pocketed to make the sheet lighter while still maintaining the load requirement (FR 5). The Alumina provides structure for the EDS electrodes and the Mylar dielectric that surrounds them.

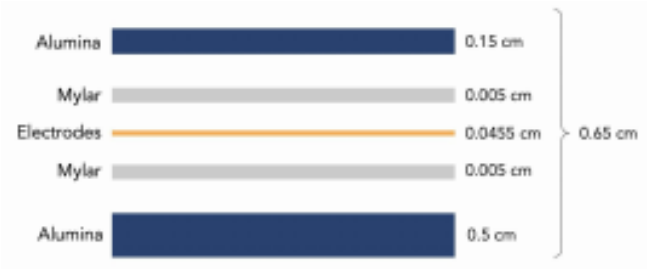

Fig. 5 Exploded view of layered panels. Figure not to scale.

The layers of the panels are held together with bolts and tapped holes. To avoid gapping, the bolts will be preloaded with a torque wrench. The total mass of each panel is $1.1 \mathrm{~kg}$, corresponding to $10.8 \mathrm{~N}$ on Earth and $1.78 \mathrm{~N}$ on the Moon.

\section{Panel Junctions}

The keys and keyways mechanically connect the tiles and provide a path for electrical connectivity. Adjacent panels are prevented from coming apart using spring-loaded ball detents. Any two tiles can be connected by matching up their edges and slotting together corresponding keys and keyways. The mechanical interface is simple and easy to attach. The ball detents built into the keyways help secure the connection against horizontal and vertical movement between panels (FR 5). Based on a consultation with astronaut Dr. Robert Behnken, tactile feedback is severely decreased when wearing spacesuit gloves. If necessary, an additional rotational locking movement can be added to provide visual and tactile feedback to the user. Since alumina is noncompliant, the mechanical connection between tiles remains rigid and ensures that electrical connections are maintained.

The EDS and power systems of each panel are connected using spring-loaded pin connectors. These pins were chosen for their resilience to mechanical shock and vibration that may come with the operation of HOMES. Springloaded pins provide a stable and low impedance connection while being compact and easy to use. In addition, spring-loaded pins have a long lifetime (FR 3). Five pinned connectors are used to facilitate rotational symmetry and provide redundancy. If testing with lunar dust simulant proves dust will penetrate the spring-loaded pin connectors, other single part spring loaded contacts will be investigated. 

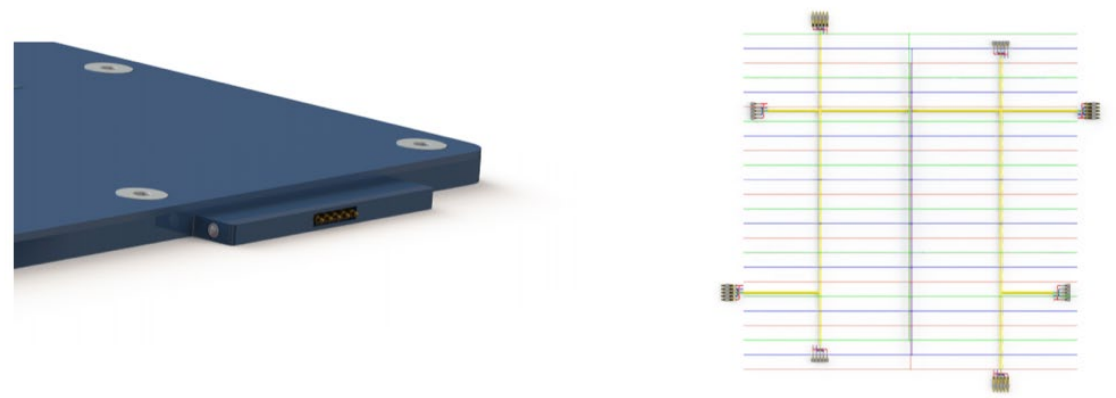

Fig. 6 An isometric view of a male finger joint (left) and a bird's eye view of the rotationally symmetric electrical connections (right).

\section{End Caps \& the Electronics Box}

End caps are necessary to insulate the exposed electrical contacts on outer panel edges. Without insulation, charged lunar dust may stick to exposed spring-loaded pins and have unintended effects (FR 1, 2). As such, the end caps do not contain any electronics or wires. The end caps act as a physical barrier to limit the amount of dust in the joints. In indoor applications, a variation of the end caps can be used to collect dust by adding a trough, for later disposal.

The electronics box is a separate module and can be placed wherever convenient. It contains the required circuitry to manage power and control modes. The box itself has a single LED, a large, centralized button, and a two-position switch. The LED displays the current mode of HOMES. The button allows the astronaut to change modes. The two-position switch changes the direction of the travelling wave generated by the power supply. As the lunar dust changes polarity through the day-night cycle, alternating the direction of the wave allows for the dust to continue traveling in the desired direction. The electronics box is connected to the panels via an integrated wire connected to an end cap. The wires are low profile and brightly colored for user safety. Both the electronics box and end caps can be seen in Fig. 7.
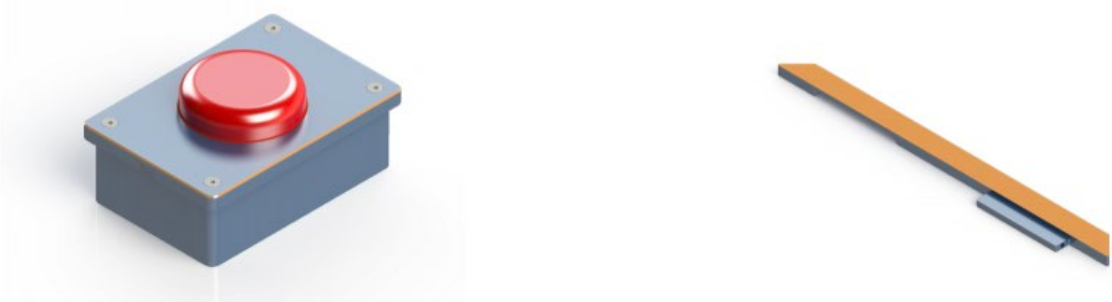

Fig. 7 Simplified electronics box (left) and end cap (right).

\section{B. Materials and Manufacturing}

The material selection is driven by the harsh lunar conditions and the functional requirements outlined at the beginning of this document. The functional temperature range and coefficient of thermal expansion properties were prioritized. Alumina was selected as the insulating material due to favorable material properties, most notably its abrasion resistant characteristics, low coefficient of thermal expansion, and wide temperature range, as noted in Table 3 below. Mylar, a space-verified material, was chosen as the dielectric for its favorable dielectric properties, similar coefficient of thermal expansion to Alumina, and wide functional temperature range. This provides sufficient structure for the EDS system while supporting the necessary electric strength and voltage applied for dust removal.

The electronics box is constructed from Aluminum 2219 [12]. With a wide temperature range $\left(-270{ }^{\circ} \mathrm{C}\right.$ to 315 ${ }^{\circ} \mathrm{C}$ ), high tensile strength, good fracture toughness, and good machinability, Aluminum 2219 is a viable option to create a secure electronics box. The electronics box will also be insulated with Multi-Layered Insulation (MLI) to reduce the heat loss by thermal radiation, as well as being hermetically sealed to protect from dust (FR 3) [13]. All materials are non-flammable. 
Table 3 Material properties.

\begin{tabular}{|c|c|c|c|c|}
\hline Metric & $\begin{array}{c}\text { Alumina (Structure) } \\
{[14]}\end{array}$ & $\begin{array}{c}316 \text { Stainless Steel } \\
\text { (Bolt) }[15]\end{array}$ & $\begin{array}{c}\text { Mylar (Dielectric) } \\
{[16]}\end{array}$ & $\begin{array}{c}\text { Aluminum } 2219 \\
{[12]}\end{array}$ \\
\hline Density & $3900\left(\mathrm{~kg} / \mathrm{m}^{3}\right)$ & $8000\left(\mathrm{~kg} / \mathrm{m}^{3}\right)$ & $1390\left(\mathrm{~kg} / \mathrm{m}^{3}\right)$ & $2840\left(\mathrm{~kg} / \mathrm{m}^{3}\right)$ \\
\hline Tensile Strength & $260(\mathrm{MPa})$ & $482(\mathrm{MPa})$ & $200(\mathrm{MPa})$ & $290 \mathrm{MPa}$ \\
\hline Flexural Strength & $379(\mathrm{MPa})$ & $290(\mathrm{MPa})$ & $2850(\mathrm{MPa})$ & - \\
\hline $\begin{array}{c}\text { Compressive } \\
\text { Strength }\end{array}$ & $2600(\mathrm{MPa})$ & $170(\mathrm{MPa})$ & - & - \\
\hline $\begin{array}{c}\text { Coefficient of } \\
\text { Thermal Expansion }\end{array}$ & $8.6 \mathrm{e}-6 /{ }^{\circ} \mathrm{C}$ & $10.3 \mathrm{e}-6 /{ }^{\circ} \mathrm{C}$ & $1.7 \mathrm{e}-5 /{ }^{\circ} \mathrm{C}$ & - \\
\hline $\begin{array}{c}\text { Dielectric Constant } \\
\text { Dielectric Strength }\end{array}$ & $9.1(\mathrm{at} 1 \mathrm{MHz})$ & - & $3.2($ at $1 \mathrm{kHz})$ & - \\
\hline Temperature Range & $-273.15^{\circ} \mathrm{C}$ to \\
& $1750{ }^{\circ} \mathrm{C}$ & $-252{ }^{\circ} \mathrm{C}$ to $871{ }^{\circ} \mathrm{C}$ & $-250{ }^{\circ} \mathrm{C}$ to $250{ }^{\circ} \mathrm{C}$ & $-253^{\circ} \mathrm{C}$ to $315^{\circ} \mathrm{C}$ \\
\hline
\end{tabular}

A 3-axis CNC machine can be used to machine the EDS grooves, connecting finger joints, end caps, and outside geometry of the alumina panels. Holes in the system can be tapped. Due to the lack of machining operations and moving parts of the system, the construction costs of HOMES will be low.

\section{Electrical Design}

\section{Circuitry and Circuit Elements}

To move dust particles, the electrodes must be powered by a 3-phase square wave, as seen in Fig. 8. Simulations showed that $3 \mathrm{kV}$ and $2.6 \mathrm{~mA}$ was sufficient to move lunar dust particles off surfaces above HOMES with a power consumption of under 8 Watts (FR 1,2). A logic circuit drives the sequence of $3 \mathrm{kV}$ square waves, which dictates the direction of the electric field. The $3 \mathrm{kV}$ are supplied by a DC-DC boost converter to increase the 28 VDC source to the required voltage. The block diagram in Fig. 8 shows the general circuit components of HOMES.

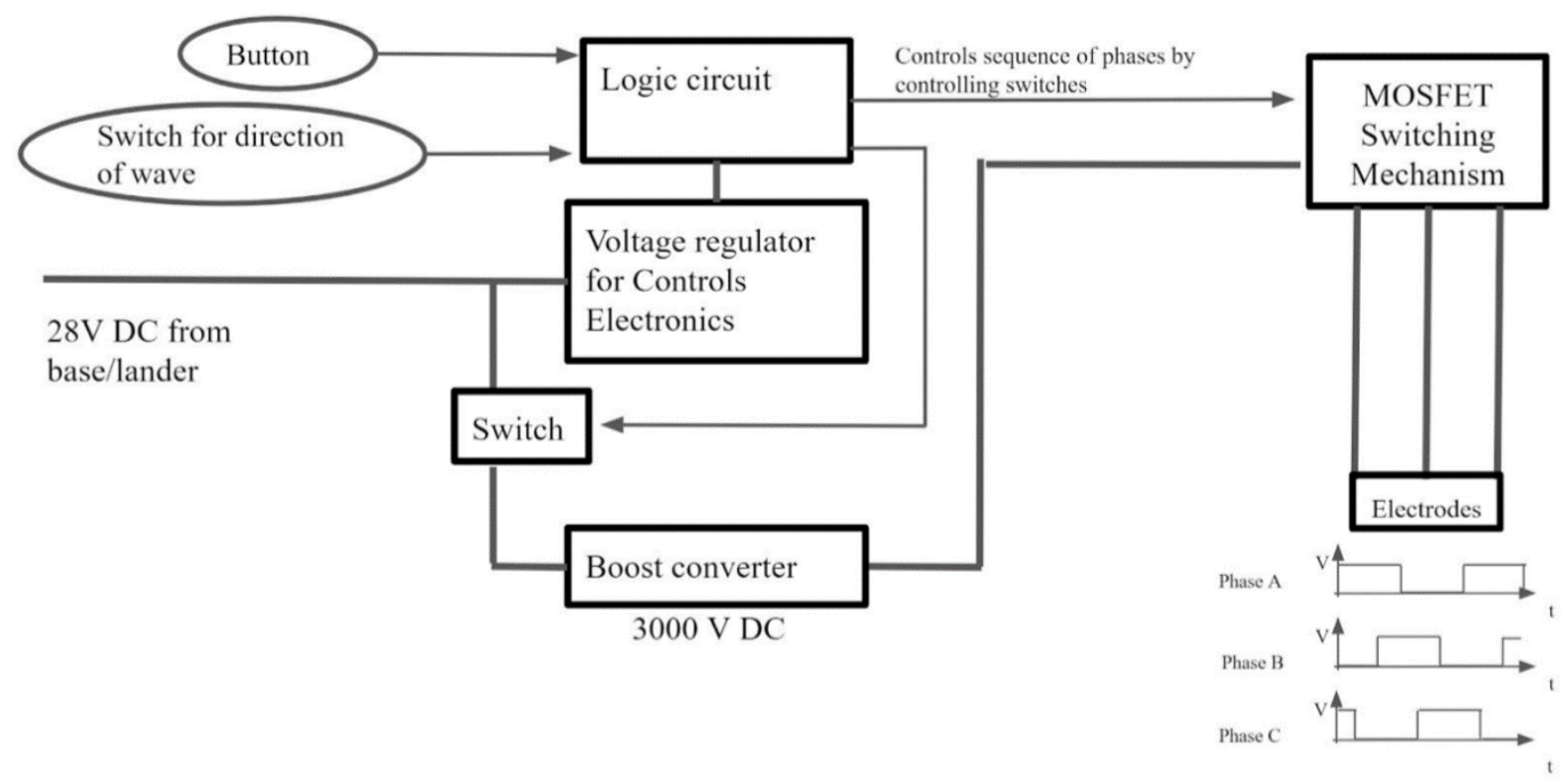

Fig. 8 Block diagram of the circuit, accompanied with an illustration of the waveforms. 
The electrodes are manipulated with n-channel enhancement mode high voltage power MOSFETs, each of them integrated with parallel diodes. Power MOSFETS are a common transistor type used in high voltage applications, including previous EDS implementations [17]. Each of the three distinct voltage phases are configured with two MOSFETs that act as switches. Two sets of MOSFETS were chosen as opposed to a singular MOSFET to handle the higher current loads (FR 1,2). Using three sets of two MOSFET switches allows for the supply voltage to be the same as the required electrode voltage, minimizing the necessary voltage on each MOSFET. The MOSFET configuration has been verified using electronic circuit simulator software (LTspice) and can be seen in Fig. 9.

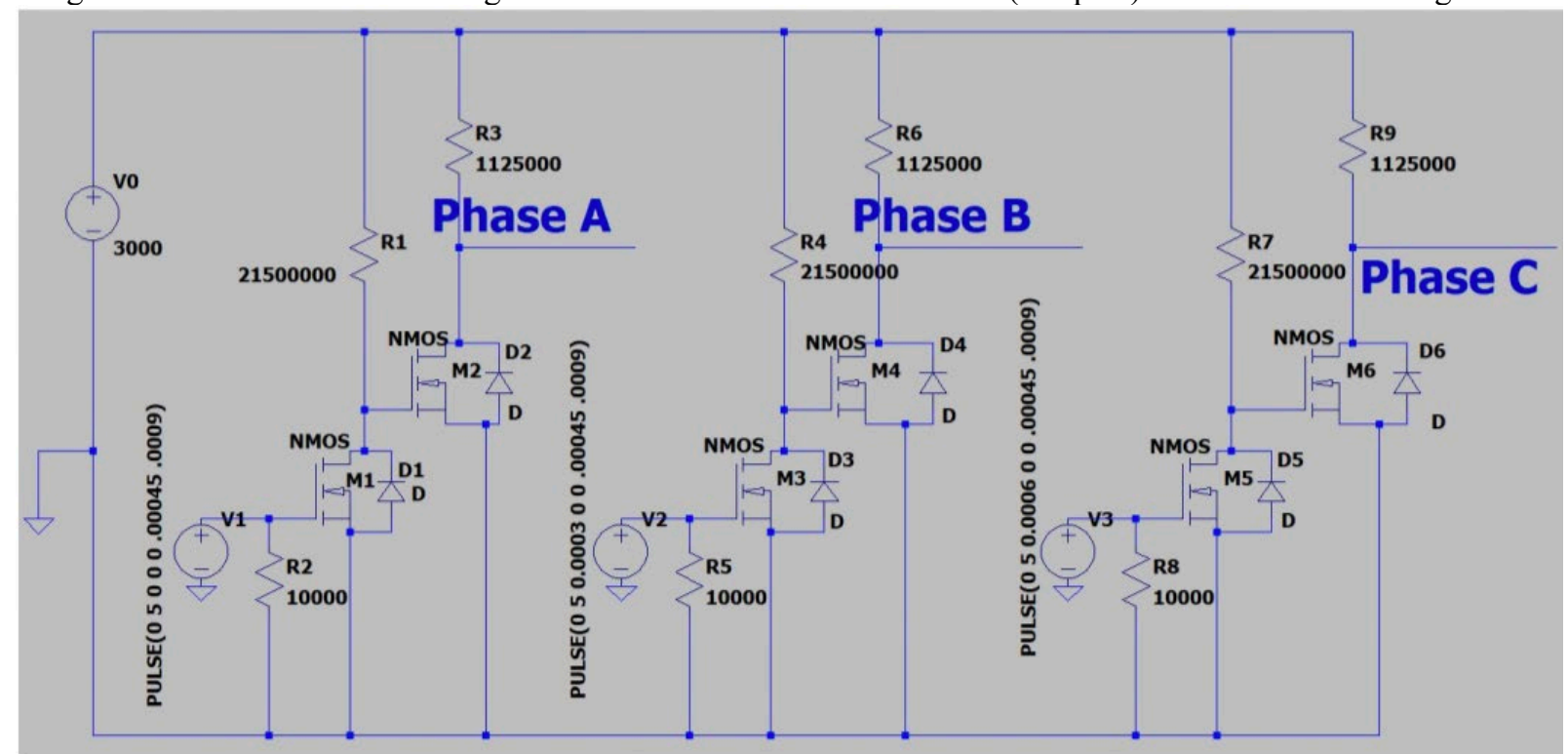

Fig. 9 Circuit diagram of MOSFET switching mechanism. The MOSFETS are located at M1, M2, M3, M4, M5, and M6. Phase A, B, and C are labeled and correspond to the phasing detailed in Fig. 8.

\section{Controls and Modes}

HOMES will have 2 functional modes, USE mode and STANDBY mode. The system will be in STANDBY mode most of the time, during which no current will be passed through the panels, and no electric fields will be generated. Astronauts will be able to switch the system into USE mode, by simply pressing a large push button attached to the electronics box, either with their hand or foot. Once in USE mode, the current will begin to run through the circuit and charges will build up on the electrodes, generating electric fields and effectively attracting lunar dust from objects placed on or near the system (FR 1,2). The system will remain in this mode until one of two things occur, either the astronaut presses the button again or a ten minute timer that began when the astronaut first pressed the button elapses, both resulting in the system returning to STANDBY mode, as seen in Fig. 10.

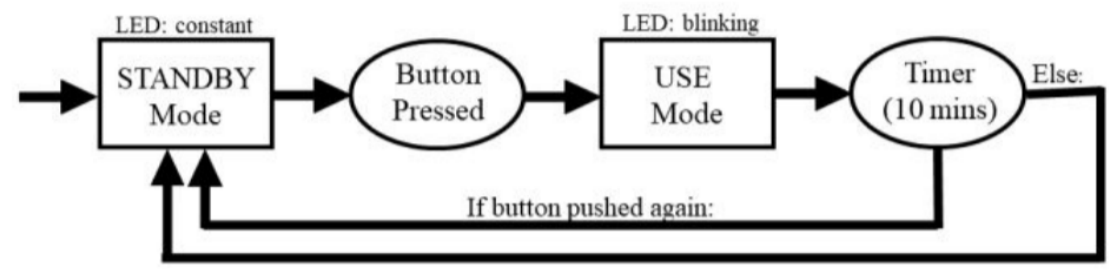

Fig. 10 Block diagram of the operational modes of HOMES.

Once USE mode is triggered, HOMES will run for ten minutes. This enables increased flexibility for astronaut use. When astronauts are using the system to clean themselves or other objects, it is likely that they will only spend a few minutes using the system (FR 1). However, if astronauts were using the system as a work surface, they would spend more time with the system in USE mode. The ten-minute interval also allows for the system to self-clean after being used. A small LED light near the electronics box will indicate to the astronauts which mode the system is in. Once the system is plugged in, it enters STANDBY mode and the light will turn on, displaying a solid light. Once USE mode is initiated, the LED will blink slowly. 


\section{Electrode Configuration}

The layout of electrodes within HOMES is designed to maximize the extent of the electric field along the top plane of each panel. To ensure that dust can seamlessly travel between multiple panels, the number of electrodes must be divisible by three, preventing like-phased wires from being adjacent. Based on a literature review of electrode pitches in EDS systems, the spacing is always between 7 and $15 \mathrm{~mm}$ [18][19]. To satisfy both of the geometry requirements, $10.4 \mathrm{~mm}$ was chosen as the electrode pitch for HOMES. The outermost electrodes are spaced $5.22 \mathrm{~mm}$, or half of the pitch, from each edge of HOMES. This electrode spacing will be optimized during initial prototyping through a combination of more rigorous simulation and physical testing. As seen on the right side of Fig. 6, each like-phased electrode shares the same color. The yellow wires between connection pins represent the power distribution wire harness feeding to every electrode. The three power distribution wires within HOMES are all twisted to negate the electric field generated by a current carrying conductor.

\section{Voltage and Power Requirements}

To gain a better understanding of the performance of HOMES, a Finite Difference Electrostatic Simulation (FDES) was conducted. The FDES was based on extensive literature on the structuring and implementation of such simulations, paying close attention to Chesnutt et al [4][18][20]. The FDES was implemented in Python and utilized the open-sourced software package MEEP [21]. An example of the FDES visualization can be seen in Fig. 11. The physics outlined in Section 3.2.2. offered key inputs into the construction of the FDES. The FDES implemented a discrete grid to spatially map electrode charge density, position, dielectric, and electric field vectors, and determine if the electric field was strong enough to overcome oppositional forces specified in equations (3) and (4). FDES determined the electric field strength and shape, given a 3-phase voltage input. The resistance and capacitance of the system was calculated based on the electrode configuration. For a single panel, the resistance is $1.2 \mathrm{M} \Omega$, determined from the resistivity of 8.6 meters of 26-gauge copper wire. The capacitance due to the Mylar dielectric in a panel is $0.81 \mathrm{pF} .3 .0 \mathrm{kV}$ square waves are consecutively applied to each of the three phases. From these values, current and power estimates were generated. The expected current and power draw are $2.6 \mathrm{~mA}$ and $8.0 \mathrm{~W}$, respectively. These estimates will be verified during operational testing but are sufficient to provide an understanding of the feasibility of the system.

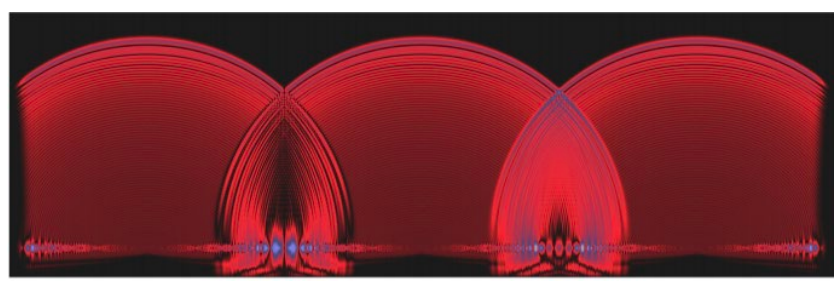

Fig. 11 Snapshot of FDES with red coloring demarcating high field intensity. The simulation captures a cross section view of three wires at the center of each circular wavefront.

\section{Use Cases and Concepts of Operations}

One of the goals of the Artemis mission is to "expand U.S. human spaceflight operations at the Moon to support sustained lunar surface activities and to demonstrate elements of a Mars-forward architecture" [22]. In order to reach this goal, astronauts will need to maintain a presence on the moon for an extended period of time. The Artemis missions will be the longest lunar missions to date [23]. Problems that have not been explicitly anticipated could arise once an extended presence on the moon is established. This is especially relevant to the effects of lunar dust on different systems on the moon, as this dust is hazardous. The Artemis missions would benefit greatly from HOMES, as the panels were designed to be as versatile as possible, lending themselves to a wide variety of use cases. The system can be configured to clean dust in a variety of directions. A few proposed arrangements and their corresponding applications are outlined below.

The lunar doormat could be a useful barrier to preventing dust from entering the habitat. Using HOMES, eight panels could be configured with strategic electric field orientations. Astronauts may place their feet on the panels and have the dust cleaned off of their boots. The dust could then be pushed off of the doormat and onto the lunar surface. Vertical panels on the exterior of the lunar habitat or lander can supplement the doormat to clean the astronaut's suit as they rotate in front of the vertical panels. Additionally, HOMES could be used to cover the floor of an airlock and could easily be configured to move all of the dust to one corner to be collected and disposed of. That way when astronauts suit up before a spacewalk or remove their suits after one, any dust that falls out of the suit could be drawn to the floor of the airlock and collected in one corner, preventing dust from making its way into 
the living space. A third application for HOMES could be a closet to store astronauts' suits, consisting of panels on the wall. That way, while the suits are not in use, they could be cleaned to reduce abrasion of the suits and prevent lunar dust from working its way further into the fabric of the suits. Again, the direction of the electric fields of the panels could easily be configured to collect the dust in one corner of the 'closet' to be disposed of. HOMES could also be placed on top of a table or other flat surface to act as a dust free work surface. The benefit of having a modular dust mitigating surface is that the direction of the electric fields generated are easily switched by removing a panel and reattaching it in a different orientation. This would be very helpful to remove dust from irregularly shaped objects and move it to the edges of the panel. Fig. 12 demonstrates some of the different applications of HOMES.

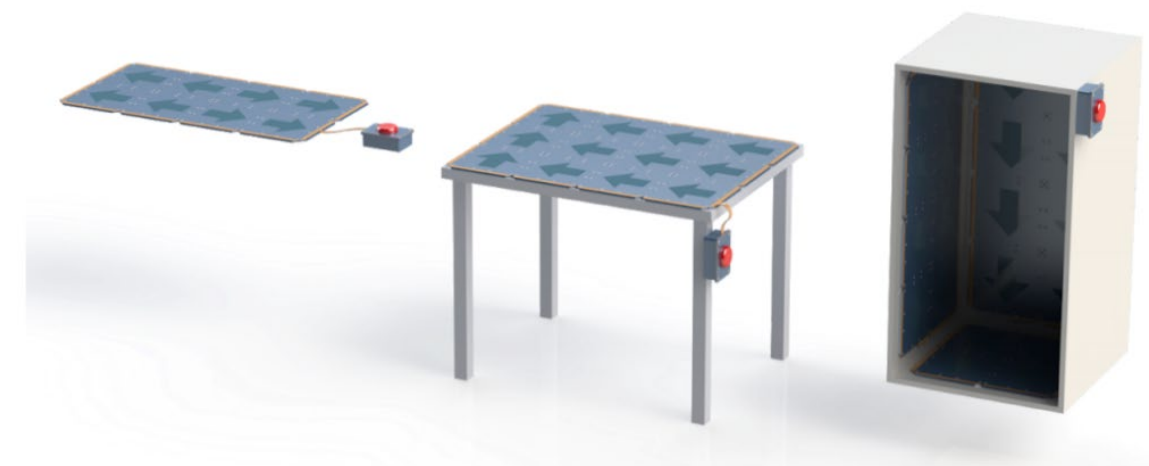

Fig. 12 Examples of different applications of HOMES. On the left is a doormat, in the center is a work surface, and on the right is a closet.

\section{Acknowledgments}

The authors would like to acknowledge the Breakthrough, Initiative, and Game-changing (BIG) Idea Challenge, the Space Grant Project, NASA, and the National Institute of Aerospace for sponsoring this research project. The authors would like to Dr. Soon-Jo Chung for his excellent advisership, guidance, and support. The authors would finally like to acknowledge Dr. Jason Kastner, Dr. Manan Arya, Dr. Charles Elachi, and Dr. Robert Behnken for support and constructive suggestions.

\section{References}

[1] Stubbs, T. J., Vondrak, R. R., \& Farrell, W. M. (2007, January). Impact of dust on lunar exploration (2007ESASP.643..239S). NASA Goddard Space Flight Center. https://www.nasa.gov/centers/johnson/pdf/486014main StubbsImpactOnExploration.4075.pdf

[2] Agenzia Spaziale Italiana (ASI), Canadian Space Agency (CSA), European Space Agency (ESA), Japan Aerospace Exploration Agency (JAXA), \& National Aeronautics and Space Administration (NASA). (2016, February). Dust mitigation gap assessment report. International Agency Working Group. https://www.globalspaceexploration.org/wordpress/docs/Dust $\% 20$ Mitigation $\% 20 \mathrm{Gap} \% 20$ Assessment $\% 20$ Report.pdf

[3] Calle, C. I., Immer, C. D., Clements, J. S., Chen, A., Buhler, C. R., Lundeen, P., Mantovani, J. G., Starnes, J. W., Michalenko, M., \& Mazumder, M. K. (2006, October). Electrodynamic Dust Shield for Surface Exploration Activities on the Moon and Mars. 57th International Astronautical Congress, Valencia, Spain.

[4] Adachi, M. (2017, February). Dynamics of Electromagnetic Particles and Its Application for Mitigation and Utilization Technologies of Regolith on Moon, Mars, and Asteroids. Waseda University. http://www.kawamoto.mech.waseda.ac.jp/kawa/pdf/2017adachi_doctor.pdf

[5] Johansen, M. R., Mackey, P. J., Hogue, M. D., Cox, R. E., Phillips, J. R., \& Calle, C. I. (2015, August). History and Flight Development of the Electrodynamic Dust Shield. AIAA Space 2015, Pasadena, CA.

[6] Guo, B., \& Javed, W. (2018). Efficiency of Electrodynamic Dust Shield at Dust Loading Levels Relevant to Solar Energy Applications. IEEE Journal of Photovoltaics, 8(1), 196-202. https://doi.org/10.1109/jphotov.2017.2764890

[7] Calle, C. I., Buhler, C. R., McFall, J. L., \& Snyder, S. J. (2009). Particle removal by electrostatic and dielectrophoretic forces for dust control during lunar exploration missions. Journal of Electrostatics, 67(2-3), 89-92. https://doi.org/10.1016/j.elstat.2009.02.012

[8] Calle, C. I., Arens, E. E., McFall, J. M., Buhler, C. R., Snyder, S. J., Geiger, J. K., Hafley, R. A., Taminger, K. M., \& Mercer, C. D. (2009). Reduced gravity flight demonstration of the Dust Shield technology for optical systems. 2009 IEEE Aerospace Conference, 1-10. 
https://doi.org/10.1109/aero.2009.4839567

[9] Buhler, C. R., Johansen, M. R., Dupuis, M., Phillips, J., Malissa, J., Wang, J., \& Calle, C. I. (2020, February). Current State of the Electrodynamic Dust Shield for Mitigation. The Impact of Lunar Dust on Human Exploration Workshop, Houston, TX.

[10] Calle, C. I., Immer, C. D., Ferreira, J., Hogue, M. D., Chen, A., Csonka, M. W., Van Suetendael, N., \& Snyder, S. J. (2010, June). Proc. ESA Annual Meeting on Electrostatics 2010, Paper D1 Integration of the Electrodynamic Dust Shield on a Lunar Habitat Demonstration Unit. ESA Annual Meeting On Electrostatics 2010.

[11] Heiken, G. H., Vaniman, D. T., \& French, B. M. (1991). Lunar Sourcebook: A User's Guide to the Moon (1st ed.). Cambridge University Press.

[12] Smiths Metal Centres. (2018). 2219 Aluminum Technical Datasheet. https://www.smithmetal.com/pdf/aluminium/2xxx/2219.pdf

[13] Fortescue, P., Swinerd, G., \& Stark, J. (2011). Spacecraft Systems Engineering (4th ed.). Wiley.

[14] Ultra-High-Temperature Alumina Ceramic. (n.d.). McMaster-Carr. Retrieved December 13, 2020, from https://www.mcmaster.com/8978T783/

[15] 316 Stainless Steel Hex Drive Flat Head Screw. (n.d.). McMaster-Carr. Retrieved December 13, 2020, from https://www.mcmaster.com/93395A348/

[16] Dupont Teijin Films. (2003, June). Mylar polyester film: Physical-Thermal properties. http://usa.dupontteijinfilms.com/wp-content/uploads/2017/01/Mylar_Physical_Properties.pdf

[17] Bock, J. P., Robison, J. R., Sharma, R., Zhange, J., \& Mazumer, M. K. (2008). An Efficient Power Management Approach for Self-Cleaning Solar Panels with Integrated Electrodynamic Screens. ESA Annual Meeting on Electrostatics.

[18] Chesnutt, J. K. W., Ashkanani, H., Guo, B., \& Wu, C.-Y. (2017). Simulation of microscale particle interactions for optimization of an electrodynamic dust shield to clean desert dust from solar panels. Solar Energy, 155, 1197-1207. https://doi.org/10.1016/j.solener.2017.07.064

[19] Toth, S. (2016, September). Electrodynamic Shielding (EDS) System to Repel Dust from Solar Concentrators. University of Florida. https://ufdcimages.uflib.ufl.edu/AA/00/05/38/74/00001/Toth UG Thesis.pdf

[20] Liu, G., Marshall, J. S., Li, S. Q., \& Yao, Q. (2010). Discrete-element method for particle capture by a body in an electrostatic field. International Journal for Numerical Methods in Engineering, 84(13), 1589-1612. https://doi.org/10.1002/nme.2953

[21] A. Oskooi, D. Roundy, M. Ibanescu, P. Bermel, J.D. Joannopoulos, and S.G. Johnson, MEEP: A flexible free-software package for electromagnetic simulations by the FDTD method, Computer Physics Communications, Vol. 181, pp. 687-702, 2010 (pdf).

[22] NASA. (2020, May). NASA's Plan for Sustained Lunar Exploration and Development. https://www.nasa.gov/sites/default/files/atoms/files/a_sustained_lunar_presence_nspc_report4220final.pdf

[23] Hollingham, R. (2019, July 19). Apollo in 50 numbers: Time. BBC Future. https://www.bbc.com/future/article/20190718apollo-in-50-numberstime\#:\%7E:text=During\%20Apollo\%2011\%2C\%20Neil\%20Armstrong,hours\%20exploring\%20the $\% 201$ unar $\% 20$ surface 